Conclusions We have developed patient-specific flow models for venous causes of PT that may be an appropriate in vitro surrogate for venous causes of PT.

Disclosures M. Amans: 1; C; NIH. 2; C; Stryker, Covidien. K. Valluru: None. H. Haraldsson: None. E. Kao: None. J. Leach: None. A. Wright: None. M. Ballweber: None. K. Meisel: None. D. Saloner: 1; C; NIH.

\section{0-026 PERSISTENT VENOUS PRESSURE GRADIENT AFTER VENOUS SINUS STENTING WITH RESOLUTION AFTER SECOND STENT PLACEMENT: A TECHNICAL NOTE}

H Dasenbrock*, A Beer-Furlan, M Chen, R Crowley. Neurosurgery, Rush University, Chicago, IL

\subsection{6/neurintsurg-2019-SNIS.26}

Object Although venous sinus stenting is an established treatment for medically refractory idiopathic intracranial hypertension (IIH), a subset of patients do not sustain improvement in symptoms post-procedurally. While many physicians perform post-stent manometry, this is not universal and there is no accepted practice regarding where pressures should be measured when pursued. In this report, two patients are described who had resolution of their pressure gradient across the stented region after venous sinus stenting, yet had a persistent physiologic venous pressure gradient elsewhere. After placement of a second stent during the same procedure, subsequent resolution of the gradient was achieved.

Methods This retrospective chart review evaluated patients at a single institution who underwent venous sinus stenting and had follow-up of at least one-year.

Results Two patients (41F and $42 \mathrm{~F}$ ) with medically refractory IIH underwent angiography with venous manometry. In the first patient, stenosis was observed at the transverse-sigmoid sinus junctions bilaterally. The maximum venous pressure was $40 \mathrm{mmHg}$, and a gradient of $30 \mathrm{mmHg}$ was present across the right transverse-sigmoid junction, where a unilateral venous sinus stent (Zilver, Cook Medical, Bloomington IN) was placed. Post-stent pressure measurements showed there was no longer a gradient from the transverse to sigmoid sinus. However, manometry in the superior sagittal sinus revealed that a significant pressure gradient persisted, which resolved after an additional stent was placed in the contralateral transverse sinus. The second patient had unilateral venous stenosis and a maximum pressure of $50 \mathrm{mmHg}$. Following placement of a right transverse-sigmoid sinus stent, the gradient was eliminated and pressures normalized in the transverse sinus. Upon measuring pressures in the superior sagittal sinus, the maximum venous pressures had only decreased to $30 \mathrm{mmHg}$, with a persistent gradient of $20 \mathrm{mmHg}$ between the superior sagittal sinus and the right transverse sinus. An additional stent was placed across this location, and the pressure gradient resolved completely. Both patients had improvement in their symptoms, which has been sustained at one-year follow-up.

Conclusions In some patients with IIH and venous sinus stenosis, placement of a single stent may eliminate the pressure gradient across the transverse-sigmoid sinus junction, yet may not sufficiently normalize pressures throughout the venous system. This may account for some patients who 'fail' venous sinus stenting. Additionally, manometry on both sides of the stent alone would not have detected a persistent pressure gradient, which was only detected with measurement in the superior sagittal sinus. We therefore advocate for measuring pressures in the superior sagittal sinus following stent placement, regardless of the location of the stent, which may detect the presence of a second clinically relevant stenosis, and an additional stent may be needed.

Disclosures H. Dasenbrock: None. A. Beer-Furlan: None. M. Chen: 2; C; Genentech, Pneumbra, Stryker, Medtronic. R. Crowley: None.

\section{0-027 MR DERIVED TTP PERFUSION MAPS ARE PREDICTIVE OF THE RATE OF STROKE EVOLUTION}

R King*, O Brooks, M Gounis, M Shazeeb. Radiology, University of Massachusetts, Worcester, MA

\subsection{6/neurintsurg-2019-SNIS.27}

Introduction Thrombectomy has rates of successful recanalization of nearly $85 \%{ }^{1}$; however, many patients may not be eligible or not have a successful outcome following recanalization if the infarct evolves prior to arriving to the angiosuite. Neuroprotection that 'freezes' the penumbra to allow a longer window to receive treatment has become the next critical topic in stroke care $^{2}$. The canine temporary large vessel occlusion model has been recently developed to allow for assessment of neuroprotectants. A limitation of this model is the rate of stroke evolution tends towards one of two pathways: fast evolution, where more than half the total infarct volume is present within 90 minutes; and slow evolution, where less than half the final volume occurs within 90 minutes. Here we present a novel analysis of MR based Timeto-Peak (TTP) maps, derived from perfusion-weighted MRI (PW-MRI), to classify the canine stroke evolution based on the first PW-MRI.

Methods Fourteen dogs were retrospectively analyzed for patterns of stroke evolution. An autologous clot was injected into the MCA in order to cause an occlusion. Once the occlusion was confirmed, the animal was moved to the MRI scanner. MRI protocol included Time of Flight (ToF), Diffusion weighted (DWI) and perfusion weighted (PWI) imaging. For PWI $0.2 \mathrm{mmol} / \mathrm{kg}$ gadopentetate dimeglumine was injected IV. To calculate the true volume of stroke, ADC maps were generated from the DWI images. The TTP maps were normalized relative to the unaffected contralateral half of the brain. The TTP map was registered to the DWI images to only account for voxels within the stroke volume. Finally, all voxels of the TTP map within the ADC lesion were binned into one of four categories: slight delay (4-8s), moderate delay (9-13s), long delay (14-18s) and extended delay ( $\geq 19 \mathrm{~s})$.

Results Using the DWI evolution as the ground truth, the canines were categorized into two groups of fast and slow evolvers (figure 1A). The binned TTP maps in the slight delay and extended delay groups also accurately predicted the evolution pathway in classifying all the canines in this study (figure $1 \mathrm{~B}$, $\mathrm{p}<0.01)$.

Conclusions The ability to predict the rate of stoke evolution, at the point of first MRI, will allow for a better 
DWI stroke evolution for fast and slow evolvers

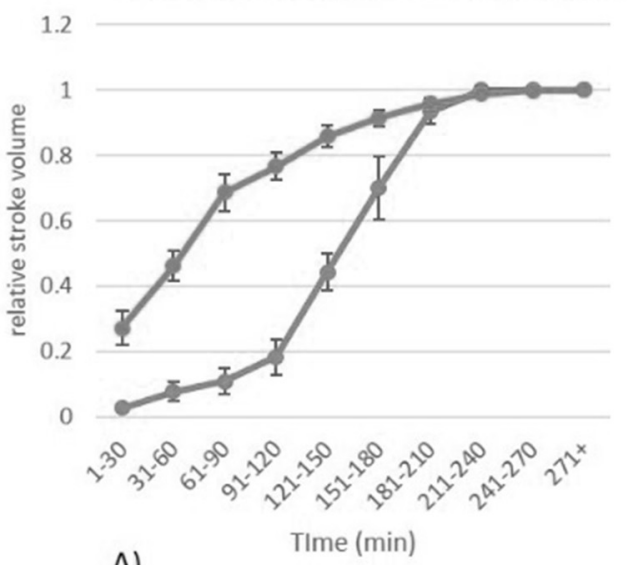

A)

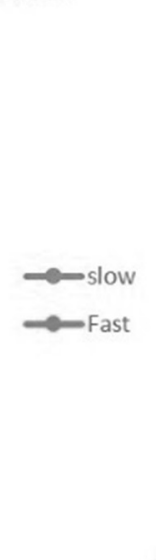

B)
Histogram binning of TTP maps

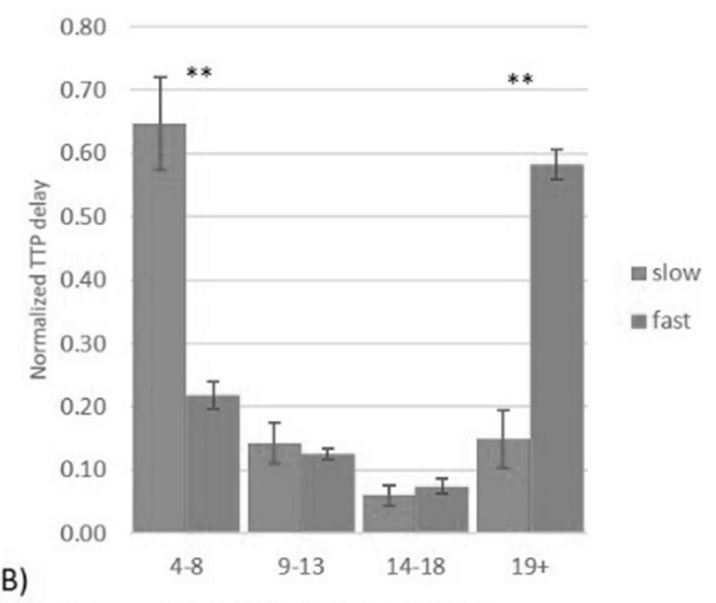

Figure: A) ADC based stroke evolution, split into two groups, fast evolvers, and slow evolvers.

B) TTP based classification of canines, predicting stroke evolution.

Abstract 0-027 Figure 1 A) ADC based stroke evolution, split into two groups, fast evolvers, and slow evolvers. B) TTP based classification of canines, predicting stroke evolution

understanding of new therapeutics. Here we have shown that by analyzing the degree of delay from the TTP map, the rate of evolution of the stroke can be predicted.

\section{REFERENCES}

1. Acute Stroke. Stroke 2017;48:1588-1593.

2. Stroke. 2011:42:3265-3270.

Disclosures R. King: None. O. Brooks: None. M. Gounis: None. M. Shazeeb: None.

\section{0-028 POST-SURGICAL PROPHYLACTIC EMBOLIZATION OF CHRONIC SUB-DURAL HEMATOMAS IN PATIENTS WITH HIGH RECURRENCE RISK: A MONOCENTRIC STUDY}

${ }^{1} \mathrm{E}$ Shotar, ${ }^{1} \mathrm{~B}$ Mathon, ${ }^{1} \mathrm{~S}$ Lenck, ${ }^{1} \mathrm{~L}$ Meyblum, ${ }^{2} \mathrm{~V}$ Degos, ${ }^{1} \mathrm{~K}$ Premat, ${ }^{1} \mathrm{~N}$ Sourour, ${ }^{3} \mathrm{~A}$ Boch, ${ }^{3} \mathrm{~A}$ Carpentier, ${ }^{1} \mathrm{~F}$ Clarençon*. 'Neuroradiology, Pitié-Salpêtrière Hospital, Paris, France; ${ }^{2}$ Neuro-Intensive Care Unit, Pitié-Salpêtrière Hospital, Paris, France; ${ }^{3}$ Neurosurgery, PitiéSalpêtrière Hospital, Paris, France

\subsection{6/neurintsurg-2019-SNIS.28}

Background and purpose The gold standard treatment for chronic subdural hematomas (cSDHs) is the surgical evacuation through a burr hole. Recurrence after such surgical procedure may occur in 10 to $20 \%$ of the cases. Embolization through the middle meningeal artery (MMA) is a promising technique for the treatment of cSDHs. The purpose of our study was to evaluate the feasibility, safety and effectiveness, in terms of recurrence reduction, of post-surgical embolization of $\mathrm{cSDH}$ in patients with a high risk of recurrence.

Materials and methods Monocentric retrospective study performed on prospectively collected data at the Pitié-Salpêtrière Hospital. From March 2018 to February 2019, embolizations with calibrated microparticles through the MMA were performed in patients surgically treated for a cSDH with a high risk of recurrence, defined as follows: 1) previous recurrence of $\mathrm{cSDH}$ or 2) antiplatelet therapy or 3) full anticoagulation therapy or 4) coagulation disorder or 5) hepathopathy or 6) chronic ethylism. In all patients, a pre-embolization supraaortic trunks (SATs) CT-angiography was performed to rule out a dumb-bell thrombus on the aortic arch or severe atheroma/tortuosity of the SATs.

Results Forty-four patients met the inclusion criteria during the inclusion period. Two patients were excluded (one in a prolonged comatose state and another with a chronic renal failure). Two patients refused the embolization procedure. A last patient was excluded due to major atheroma on the SATs. Finally, 39 patients with 43 cSDHs (4 patients had bilateral SDHs) underwent the embolization procedure. Thirty-seven embolization procedures (95\%) were performed under local anesthesia. Among the 43 cSDHs, 5 (9\%) could not be embolized due to catheterization failure ( 4 cases) or to the presence of a 'dangerous anastomosis' (1 case). No complication (either major or minor) was recorded. Only one recurrence $(2.6 \%)$ requiring a surgical retreatment was recorded during the follow-up period.

Conclusion Post-surgical embolization through the MMA is a simple and safe procedure, which may reduce the recurrence risk of cSDHs. These preliminary results should be confirmed by randomized controlled trials.

Disclosures E. Shotar: None. B. Mathon: None. S. Lenck: None. L. Meyblum: None. V. Degos: None. K. Premat: None. N. Sourour: 2; C; Medtronic. A. Boch: None. A. Carpentier: None. F. Clarençon: 2; C; Balt, Artedrone, Penumbra.

\section{0-029 BRAIN TISSUE IRON QUANTIFICATION AT THE PERIPHERY OF THE HEMATOMA BY MRI IN PATIENTS WITH INTRACEREBRAL HEMORRHAGE: TRANSLATIONAL EVIDENCE IN FIRST 10 PATIENTS}

${ }^{1} \mathrm{~N}$ Chaudhary*, ${ }^{2} \mathrm{~A}$ Pandey, ${ }^{1} \mathrm{~J}$ Griauzde, ${ }^{1} \mathrm{G}$ Gemmete, ${ }^{2} \mathrm{R}$ Keep, ${ }^{2} \mathrm{G}$ Xi. ${ }^{1}$ Radiology, University of Michigan Health System, Ann Arbor, Ml; ${ }^{2}$ Neurosurgery, University of Michigan Health System, Ann Arbor, MI

\subsection{6/neurintsurg-2019-SNIS.29}

Objective Intracerebral hemorrhage (ICH) has devastating consequences in the human population. No specific outcome modifying treatment or objective risk stratifying measure currently exists in ICH patients. The goal of our study is to 